\title{
Genetic diagnosis of patients with esophageal cancer using FISH
}

\author{
IDIRIS AWUT ${ }^{1}$, MADINIYET NIYAZ $^{2}$, XIE HUIZHONG ${ }^{3}$, HADETI BIEKEMITOUFU ${ }^{4}$, ZHANG HONG YAN ${ }^{5}$, \\ ZHANG ZHU $^{1}$, ILYAR SHEYHEDIN ${ }^{1}$, ZHANG CHANGMIN ${ }^{1}$, ZHANGLI WEI ${ }^{1}$ and WEN HAO $^{2}$ \\ ${ }^{1}$ Thoracic Surgery, ${ }^{2}$ Xinjiang Esophageal Cancer Research Institute, Medical Research Center, \\ Departments of ${ }^{3}$ Gastroenterology and ${ }^{4}$ Pathology, and ${ }^{5}$ Endoscopy Center, \\ First Affiliated Hospital of Xinjiang Medical University, Xinjiang 830054, P.R. China
}

Received April 29, 2010; Accepted June 21, 2010

DOI: 10.3892/ol_00000142

\begin{abstract}
This study aimed to the clarify the diagnostic efficacy of fluorescence in situ hybridization (FISH) in Kazakh patients with esophageal cancer (EC). FISH was compared with the pathological examination of biopsy specimens with DNA probes. We enrolled 20 patients, of which 15 were males and 5 females, with an average age of 58.3 years, who had abnormal esophaguses on barium radiological digital imaging. Touch preparations were performed on biopsy specimens from all of the patients and were examined using FISH for chromosomal abnormalities. We compared the FISH results with the pathology slides stained with hematoxylin and eosin. Classification, according to pathology, identified 2 cases of class II, 3 cases of IIIa, 1 case of IIIb, 2 cases of IV, 12 cases of class V and no cases of class I. The cases classified as class IIIb or higher were considered to be positive for cancer. Using histopathology, 10 cases were diagnosed with squamous cell carcinoma and 5 were diagnosed as adenocarcinoma, with one case being false-negative. Thus, the sensitivity of the pathological examination was $93 \%$ and the specificity was $100 \%$. Using FISH, 16 cases showed aberrant copy numbers in either chromosome 3 or 17. By comparison, pathology did not reveal any false-positive or false-negative cases with a sensitivity and specificity of $100 \%$. The centromeres of chromosome 3 copy numbers was significantly higher $(\mathrm{p}=0.035)$ than the centromeres of chromosome 17. Our study compared FISH to diagnose aneusomic esophageal cancer cells with the pathology of biopsied tissue. Our findings suggest that FISH
\end{abstract}

Correspondence to: Dr Wen Hao, Xinjiang Esophageal Cancer Research Institute, Medical Research Center, First Affiliated Hospital of Xinjiang Medical University, 137 Liyushan South Road, Urumqi, Xinjiang 830054, P.R. China

E-mail: dr.wenhao@163.com

Abbreviations: FISH, fluorescence in situ hybridization; EC, esophageal cancer; SSC, standard saline citrate

Key words: aneusomy, fluorescence in situ hybridization, pathology, esophageal cancer is a useful and objective assay for the detection of malignant cells of esophageal cancer. In our study, the centromeres of chromosome 3 was the more sensitive probe for the diagnosis of esophageal cancer in Kazakh patients.

\section{Introduction}

The incidence of squamous cell carcinoma of the esophagus and gastroesophageal junction cancer has markedly increased over the past 30 years in Kazakh people living in Xinjiang, west of China (1). These types of cancer are associated with a poor prognosis unless detected and treated while in the early stages. Conventional pathologic methods usually play an important role in the diagnosis of cancer by providing important differential information between benign and malignant diseases $(2,3)$. However, pathological evaluation is occasionally only suggestive and not conclusive. This may lead to a conflict between pathology and clinical diagnoses. These conflicting findings have stimulated the search for more objective and quantitative methods for the diagnoses of the related cancer.

Aneuploidy is the most common feature of many solid tumors (4), including esophageal cancer (EC). Solid tumors are characterized by complicated karyotypes using classical genetic parameters $(5,6)$. Thus, malignant cells can be diagnosed by detecting aneuploidy usually found in aneusomic nuclei. A rapid and sensitive method for detecting the aneusomy of a specific chromosome in an individual cell is fluorescence in situ hybridization (FISH) (7-9). For this purpose, specific centromeric DNA probes have enumerated the degrees of aneuploidy.

FISH was originally developed as a method to detect chromosome aberrations and is now widely used for gene mapping for the diagnosis of congenital diseases and for detecting specific gene copy numbers found in malignant cells (4,9-11).

One advantage of the FISH method is that it is an objective and quantitative evaluation procedure for the detection of malignant cells. However, the sensitivity and specificity in the diagnosis of EC in Kazakh people have yet to be determined. We report the results of an original study in which we compare the FISH procedure with clinical findings and conventional pathological methods to detect esophageal cancer cells. 


\section{Patients and methods}

From January 2009 to January 2010, 20 patients with suspected esophageal cancer were tested by barium radiological digital imaging. Biopsy material was further examined pathologically at the First Affiliated Hospital of Xinjiang Medical University. The results confirmed that all of the patients had esophageal cancer and thus were enrolled in this study. The patients included 15 males and 5 females, with an average age of 58.3 years (range 45-75).

Tissues appearing to be suspicious of neoplasms were biopsied by fiberoptic esophageal-gastroscopy. These samples were tested and analyzed by pathology and FISH. Informed consent was provided by all of the patients.

Each biopsied lesion was treated in duplicate and subjected to five touch impressions on sterile slides for FISH analysis and then preserved in formalin for later pathological examination.

For pathology, cells were stained with hematoxylin and eosin. Pathologic evaluations were carried out by pathologists in the Department of Pathology at the First Affiliated Hospital of Xinjiang Medical University.

The histopathology of the biopsies was classified as follows: class I indicated the absence of atypical or abnormal cells; class II indicated atypical pathology with no evidence of malignancy; class III was suggestive of, but not conclusive for, malignancy; IIIa indicated mild dysplasia albeit not cancerous; IIIb indicated advanced dysplasia; class IV was strongly suggestive of malignancy; and class $\mathrm{V}$ was indicative of malignancy.

For FISH, dual color probes were prepared. Briefly, touch preparations of cells were made on glass slides, air-dried overnight at $25^{\circ} \mathrm{C}$ and then stored at $-80^{\circ} \mathrm{C}$. Centromeric probes labeled with fluorochrome were used for the visualization and enumeration of copy numbers. Spectrum orange- and greenlabeled probes were used to visualize centromeric regions of chromosomes 3 and 17. Reagents were purchased from Abbott Molecular, Inc., Des Plaines, IL, USA.

Frozen touch preparations of cells were denatured with $70 \%$ formamide and then washed twice in standard saline citrate (SSC) at $74^{\circ} \mathrm{C}$ and at $25^{\circ} \mathrm{C}$ each for $2 \mathrm{~min}$ in a water bath. Slides were then dehydrated through a series of graded ethanol dilutions (70, 85 and $100 \%$, each for $2 \mathrm{~min}$ ). A hybridization solution $(10 \mu \mathrm{l})$ was applied to each microscope slide. The hybridization solution contained $1 \mu \mathrm{l}$ of each of the DNA probes, $7 \mu \mathrm{l}$ of a hybridization buffer and $1 \mu \mathrm{l}$ of double distilled water. The solution was covered with a coverslip and sealed with rubber cement. After incubation for $16 \mathrm{~h}$ at $42^{\circ} \mathrm{C}$ in a humidity chamber, slides were washed with an SSC solution for $5 \mathrm{~min}$ at $74^{\circ} \mathrm{C}$ and $25^{\circ} \mathrm{C}$ for $2 \mathrm{~min}$. Diamidinophenylindole II (an anti-face solution; $5 \mu \mathrm{l}$ ), was applied to each spot and covered with a coverslip. The slides were observed under a fluorescence microscope which was connected to a cooled charge-coupled device camera and an image analyzer system (Leica Microsystems, Ltd., Germany).

FISH signal analysis was then carried out. All cells, apart from damaged cells or those with overlapping nuclei, were evaluated. A total of 100 nuclei were counted, and the total numbers of centromeric signals were recorded. If there were $<100$ nuclei on a slide, as many nuclei as possible were counted and enumerated for centromeric signals. When the percentage
Table I. Clinical features of the patients.

\begin{tabular}{cccc}
\hline Case & Age & Gender & Histology \\
\hline 1 & 71 & $\mathrm{M}$ & $\mathrm{Sq}$ \\
2 & 52 & $\mathrm{~F}$ & $\mathrm{Ad}$ \\
3 & 45 & $\mathrm{M}$ & $\mathrm{Ad}$ \\
4 & 70 & $\mathrm{M}$ & $\mathrm{Ad}$ \\
5 & 53 & $\mathrm{M}$ & $\mathrm{Neg}$ \\
6 & 75 & $\mathrm{M}$ & $\mathrm{Neg}$ \\
7 & 45 & $\mathrm{~F}$ & $\mathrm{Sq}$ \\
8 & 71 & $\mathrm{M}$ & $\mathrm{Sq}$ \\
9 & 55 & $\mathrm{M}$ & $\mathrm{Sq}$ \\
10 & 58 & $\mathrm{M}$ & $\mathrm{Sq}$ \\
11 & 54 & $\mathrm{~F}$ & $\mathrm{Sq}$ \\
12 & 69 & $\mathrm{M}$ & $\mathrm{Sq}$ \\
13 & 55 & $\mathrm{M}$ & $\mathrm{Neg}$ \\
14 & 54 & $\mathrm{M}$ & $\mathrm{Ad}$ \\
15 & 62 & $\mathrm{M}$ & $\mathrm{Sq}$ \\
16 & 56 & $\mathrm{~F}$ & $\mathrm{Neg}$ \\
17 & 45 & $\mathrm{~F}$ & $\mathrm{Sq}$ \\
18 & 62 & $\mathrm{M}$ & $\mathrm{Neg}$ \\
19 & 54 & $\mathrm{M}$ & $\mathrm{Ad}$ \\
20 & 60 & $\mathrm{M}$ & $\mathrm{Sq}$ \\
\hline
\end{tabular}

M, male; F, female; Ad, adenocarcinoma; Sq, squamous cell carcinoma; Neg, negative case.

of hyperdisomic nuclei (i.e., more than three copies for at least one nucleus) was $>10 \%$, we judged the tissue to be malignant.

FISH diagnosis was made without clinical information or the result of conventional histopathology, nor were the results of FISH analysis made known to the pathologists. Thus, the two diagnoses were independently performed in a blinded manner.

The $\mathrm{t}$-test was used to determine the significance between the number of countable copy number centromeres in chromosomes 3 and 17. $P<0.05$ was considered to indicate a statistically significant difference.

\section{Results}

Specific diagnoses made based on histopathology determined that 15 patients had primary esophageal cancer, 10 cases had squamous cell carcinoma, 5 cases had adenocarcinoma and 5 cases had benign lesions (Table I).

Classification was as follows: 2 cases were class II, 3 cases were IIIa; 1 case was IIIb; 2 cases were class IV; 12 cases were class V; and no cases were class I. Classes higher than IIIb were positive for esophageal cancer. One case was false-negative, but no cases were false-positive. Thus, using histopathology as a parameter, sensitivity was $93 \%$ and specificity was $100 \%$.

FISH analyses (30-100 countable nuclei) showed that 16 of the 20 cases had abnormal copy numbers in either chromosome 3 or 17. Representative findings comparing biopsy pathology and FISH are shown in Fig. 1.

FISH did not reveal any false-positive or false-negative cases with a sensitivity and specificity of $100 \%$ (Table II). The 
Table II. Results of FISH and conventional biopsy pathology.

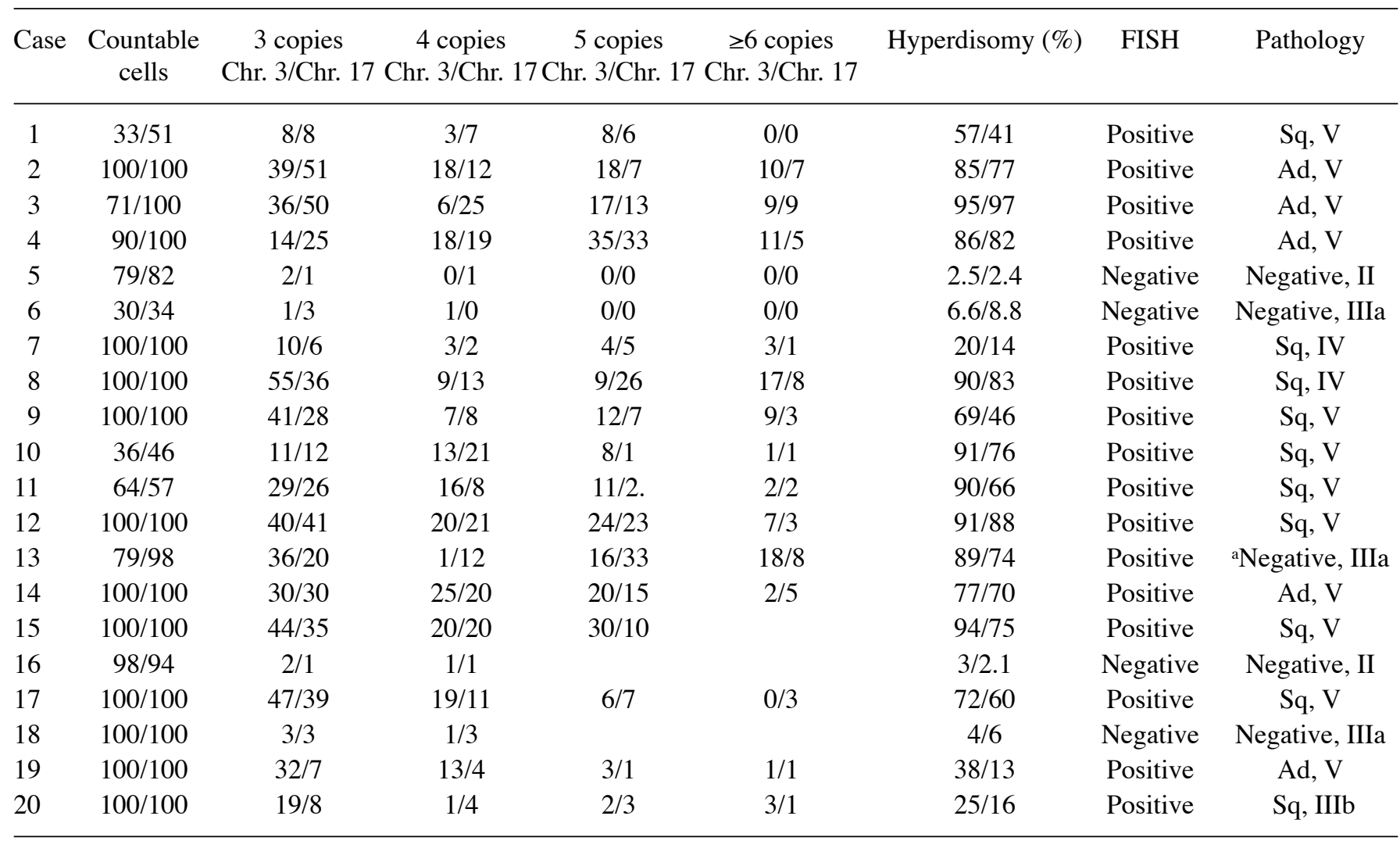

Chr, chromosome; FISH, fluorescence in situ hybridization; Sq, squamous cell carcinoma; Ad, adenocarcinoma; II-V, grade. ${ }^{\mathrm{a}}$ False-negative.

centromeres of chromosome 3 copy numbers were significantly higher $(\mathrm{p}=0.035)$ than those of chromosome 17 .

\section{Discussion}

We demonstrated that FISH analysis is more accurate in diagnosing esophageal cancer than using pathology examinations of biopsies. Similar results using FISH analyses were reported in a number of studies $(4,8,10,12)$. However, our findings contrasted FISH with those of the pathology of the biopsy specimens. Han et al (13) examined 113 EC patients using multiplex FISH with chromosome-specific centromere DNA probes $3,8,10,12,17$ and 20 . In that study, chromosomal signal numbers and all of the chromosomes were found to be abnormal copy numbers. The present study used only two probes which were specific for the centromeric region of chromosomes 3 and 17.

Fritcher et al (6) investigated esophageal adenocarcinoma using the FISH method with centromeric region probes C-MYC, P16, HER2 and 20q13. In that study, the sensitivity of cytology was $45 \%$ for the detection of esophageal adenocarcinoma, but FISH was $100 \%$. The same study used FISH analysis with centromeric probes 7,11,12, 17 and 18. Aneusomy was not found in the normal controls of any chromosomes. In contrast, chromosomal abnormalities were found in all carcinoma specimens (14). In these studies, the FISH method detected esophageal squamous cancer and adenocarcinoma cells in touch preparations of resected tumors. Thus, our study compared the pathology of biopsy specimens to the FISH method.
The cut-off value for the percentage of hyperdisomic cells was set at $10 \%$ (i.e., more than $10 \%$ means cancer is present and less than $10 \%$ means absence of cancer), since normal cells are often less than $6 \%$. This discrepancy probably occurs due to the counting of sister chromatids as copies. When we set the cut-off value at $10 \%$, no false-positive or false-negative cases were noted. Additionally, the sensitivity and specificity was $100 \%$. Centromeres of chromosome 3 copy numbers were significantly higher than those of chromosome 17 . The reason for this discrepancy is unknown but Kazakh people have a higher incidence of cancer (1) which may correlate with their higher copy numbers in chromosome 3 .

Using biopsy pathology we found one false-negative case. In this case, barium radiological digital imaging and examination by esophageal gastroscopy strongly suggested cancer; the clinical diagnosis was recurrent $\mathrm{EC}$, but the pathologic diagnosis was IIIa. These findings suggest that FISH is able to detect chromosome aberrations that may lead to the commencement of oncogenesis of EC (i.e., IIIb). This hypothesis has been supported by other studies $(6,13)$. Additional studies are needed to further evaluate and support our findings. For example, we propose using different ethnic groups and increased numbers of patients. We believe that FISH may provide decisive information for the detection of malignancies, particularly in cases classified as IIIa or IIIb. Subsequently, the FISH assay may have a higher sensitivity of diagnosis than other current methods.

In a previous study, we did not correctly diagnose malignancy in lung cancer patients using FISH $(15,16)$. There are 

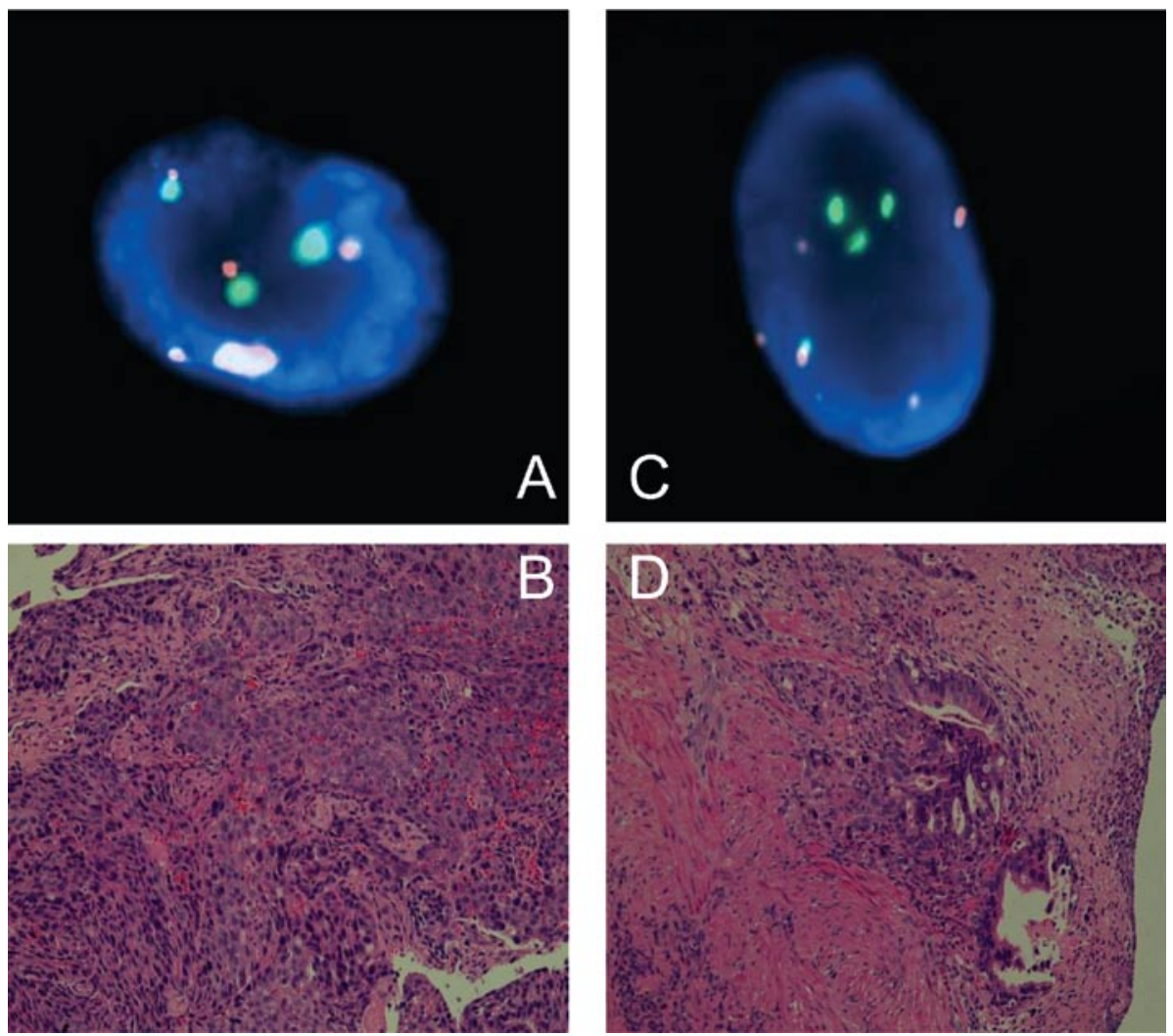

Figure 1. FISH analysis of the centromere of chromosome 3 (red signals) and chromosome 17 (green signals). Normal representative nuclei carry 2 copies of the centromeres of chromosomes 3 and 17. (A and B) Pathology results indicate grade V squamous cell carcinoma (case 15). FISH representative nuclei carrying 5 copies of the centromere of chromosome 3 and 3 copies of the centromere of chromosome 17. H\&E staining; magnification, $x 400$. (C and D) Pathology results indicate grade V adenocarcinoma (case 19). FISH representative nuclei carrying 6 copies of the centromere of chromosome 3 and 4 copies of the centromere of chromosome 17. H\&E staining; magnification, $\mathrm{x} 400$.

two possible reasons for our false-negative FISH results. One would be the failure to obtain cell material that was absent in cancer cells. The second reason would be that the cancer cells were near-diploid. Therefore, we were not able to detect aneusomy in the target chromosomes.

Previous studies suggest that the sensitivity of FISH is superior to that of conventional cytology $(13,16)$. However, there are some disadvantages to the FISH method of analysis. To begin with, this method does not generate information about the histopathology or type of esophageal cancer since one cannot observe morphological features. Furthermore, FISH is expensive. A further drawback is that FISH signal counting under fluorescence microscopy is time-consuming and requires trained personnel.

In the future, we expect to be able to detect more aneusomic cells using additional probes for other chromosomal regions as reported (17-19), e.g., EGFR, C-MYC, HER2, P16, P53, cyclin D1, X and Y, which may be successful.

Taken together, FISH can detect multiple chromosomal changes and is an ideal prognostic tool, thereby opening the door to different therapeutic approaches in the future (18).

In conclusion, the FISH method successfully detected esophageal cancer in Kazakh patients using only two probes. This method appeared more sensitive than the biopsy pathology diagnostic method. Centromeres of chromosome 3 showed the most sensitive probe diagnostically in Kazakh patients with esophageal cancer.

\section{Acknowledgements}

This work was supported by a grant from the First Hospital of Xinjiang Medical University (2008-YFY-06) and the Chinese Postdoctoral Fund of Xinjiang Medical University (200803014). Thanks are extended to the Department of Hematology, First Affiliated Hospital of Xinjiang Medical University for the technical support. We thank Professor Glenn S. Bulmer for reviewing this manuscript.

\section{References}

1. Zhang YM: Distribution of esophageal cancer in the Xinjiang. XMU Med 11: 139-145, 1988.

2. Odze RD: Barrett esophagus: histology and pathology for the clinician. Nat Rev Gastroenterol Hepatol 6: 478-490, 2009.

3. Yerian L: Histology of metaplasia and dysplasia in Barrett's esophagus. Surg Oncol Clin N Am 18: 411-422, 2009.

4. Rajagopalan $\mathrm{H}$ and Lengauer C: Aneuploidy and cancer. Nature 432: 338-341, 2004.

5. Halling KC and Kipp BR: Fluorescence in situ hybridization in diagnostic cytology. Hum Pathol 38: 1137-1144, 2007.

6. Fritcher EG, Brankley SM, Kipp BR, et al: A comparison of conventional cytology, DNA ploidy analysis, and fluorescence in situ hybridization for the detection of dysplasia and adenocarcinoma in patients with Barrett's esophagus. Hum Pathol 39: 1128-1135, 2008.

7. Doak SH, Jenkins GJS, Parry EM, Griffiths AP, Shah V, Baxter JN and Parry JM: Characterisation of p53 status at the gene, chromosomal and protein levels in oesophageal adenocarcinoma. Br J Cancer 89: 1729-1735, 2003. 
8. Nakamura H, Saji H, Idiris A, et al: Chromosomal instability detected by fluorescence in situ hybridization in surgical specimens of non-small cell lung cancer is associated with poor survival. Clin Cancer Res 9: 2294-2299, 2003.

9. Dey P: Aneuploidy and malignancy: an unsolved equation. J Clin Pathol 57: 1245-1249, 2004.

10. Albertson DG, Collins C and McCormick F: Chromosome aberrations in solid tumors. Nat Genet 34: 369-376, 2003.

11. Bahmanyar S and Ye W: Dietary patterns and risk of squamouscell carcinoma and adenocarcinoma of the esophagus and adenocarcinoma of the gastric cardia: a population-based casecontrol study in Sweden. Nutr Cancer 54: 171-178, 2006.

12. Varshney D, Zhou YY, Geller SA and Alsabeh R: Determination of HER-2 status and chromosome 17 polysomy in breast carcinomas comparing Hercep Test and PathVysion FISH assay. Am J Clin Pathol 121: 70-77, 2004.

13. Han QY, Shun H, Yu PW, et al: [Application of multicolor fluorescence in situ hybridization to early diagnosis of esophageal squamous cell carcinoma]. Ai Zheng 27: 396-401, 2008.

14 You XY, Johannes F, Shuang LX and Chiang JL: Genomic instability in precancerous lesions before inactivation of tumor suppressors p53 and APC in patients. Cell Cycle 13: 1443-1447, 2006.
15. Idiris A, Nakamura $\mathrm{H}$, Maidiniyeti $\mathrm{N}$, et al: Detection of lung cancer cells in bronchial washings using fluorescence in situ hybridization. J Jpn Bronchoesophagol Soc 56: 470-475, 2005.

16. Nakamura H, Idiris A, Kawasaki N, et al: Quantitative detection of lung cancer cells by fluorescence in situ hybridization: comparison with conventional cytology. Chest 128: 906-911, 2005.

17. Reichelt U, Duesedau P, Tsourlakis M, et al: Frequent homogeneous HER-2 amplification in primary and metastatic adenocarcinoma of the esophagus. Mod Pathol 20: 120-129, 2007.

18. Arnold D, Peinert S, Voigt W and Schmoll HJ: Epidermal growth factor receptor tyrosine kinase inhibitors: present and future role in gastrointestinal cancer treatment: a review. Oncologist 11: 602-611, 2006

19. Kawaguchi Y, Kono K, Mimura K, Mitsui F, Sugai H, Akaike H and Fujii H: Targeting EGFR and HER-2 with cetuximab and trastuzumab-mediated immunotherapy in esophageal squamous cell carcinoma. Br J Cancer 97: 494-501, 2007. 